\title{
Population Health: Determinants and Interventions
}

\author{
Nancy Edwards, RN, PhD
}

The population health concept has been identified as a major component of the new Health for All Strategy for the 21st Century. ${ }^{1}$ This new policy follows two decades of efforts to achieve the objectives outlined in the Alma Ata Declaration. During this period of Canadian public health history, we have seen a shift in the focus of community health services from interventions targeting lifestyle change to programming which embraces the tenets of health promotion. More recently, population health has gained prominence as an underlying concept for public health programs. ${ }^{2}$ Building on the experience and knowledge gained from lifestyle and health promotion efforts, population health focusses our attention on inequalities in health status and their determinants. As we enter the new millennium, a major challenge facing those who design, manage and implement public health programs will be finding the means to effectively tackle determinants and their interactions.

Determinants can be understood as causes, mediating factors and contextual influences. A great deal of research has focussed on determinants as causes. Medical and epidemiological sciences for example, aim to discover the etiology of disease. Unravelling these causes requires a clear understanding of the mechanism(s) through which a single agent, a set of factors, or a sequence of circumstances can lead to a disease or illness. Study findings in the epidemiological literature are often based on models examining relationships between risk factors and disease. These models shed some light on characteristics which appear to protect against disease. However, a more expansive understanding of what protects against disease or illness might emerge from models examining causal links between determinants and

School of Nursing, Faculty of Health Sciences, University of Ottawa, Ottawa, ON health, rather than determinants and disease. This "reverse" causal thinking would identify additional study variables to measure and new statistical models to test. Theoretical models (e.g., models of resilience, capacity development, meaningful participation in society, and social cohesion) are required to guide this type of thinking.

While great strides have been made in discovering biological mechanisms for disease, interactions between social and genetic determinants, and between social and physical environmental determinants are more perplexing. For example, although interactions resulting from the combined effects of physical environmental exposures such as asbestos and cigarette smoke have been well documented, we are only beginning to understand the synergistic impact of poverty, poor social support, and tobacco use. The study of paradoxes, defined as observations which contradict our current understanding of cause and effect relationships, ${ }^{3}$ encourages us to question assumptions about at risk populations. For example, what are the factors that build resilience among youth living in high risk families or communities? Why are low birthweight rates much lower in some socio-economically disadvantaged groups than others?

Mediating determinants are those which diminish, enhance or modify the relationship between two or more factors. These determinants can create the conditions necessary for interactions. Determinants can also influence processes of change from one state to another. Increasingly, transitions through the life cycle are viewed as being critical intervention points. There is much to be learned about determinants which ease these transitions or make them more difficult.

Context concerns the setting, the situation, or the scenario wherein determinants act as causes or mediators. At the macro end of the scale, contextual influences are broad system-level factors such as political influences (e.g., change in government), and global economic forces (e.g., crash of the Asian market). These influences are generally more subtle in our daily life. However, they are a constant force, shaping our values, our opinions, and our expectations. At the other end of the spectrum, micro-level determinants are those which consciously influence us day-to-day. For example, the settings where we spend our time (workplace, home) and our social contacts in those settings shape our health and illness experiences. In their critique of social determinants as conceptualized by the Canadian Institute of Advanced Research, Poland et al. ${ }^{4}$ argue that the broader social and political forces for determinants of health (e.g., globalization and class structure) warrant a more complete analysis.

Ultimately, research on determinants is intended to guide the development of interventions. With a deeper understanding of determinants, their interactions, and contextual influences, the sophistication of public health programs and their subsequent impact should improve. However, the challenge of applying a better understanding of determinants lies in their complexity and the fact that they are dynamic, creating constantly changing conditions for interventions. Thus, a determinants perspective points to the need for multicomponent programs designed with an intent to build potential synergies among intervention strategies. For the program planner, this raises many questions about the optimal combinations and sequences of multiple interventions; the relative contributions of component interventions; the impact of frequency, intensity, interdependence, and differentiation; and the iterative nature of multiple intervention impact (in series, in parallel and with lag times). New decision-support tools and strategies will 
be needed to help program managers deal with this unprecedented level of complexity in program design. ${ }^{5}$ This is truly the challenge of population health; how to use the emerging evidence about determinants and their interactions to guide development of the next generation of public health programs.

\section{REFERENCES/BIBLIOGRAPHIE}

1. Zollner H, Lessof S. Population Health - Putting Concepts into Action. Final Report. World Health Organization, Regional Office for Europe. August, 1998.

2. Hayes MV, Dunn JR. Population Health in Canada: A Systematic Review. Canadian Policy Research Networks Inc., 1998.

3. Guttman N, Kegler M, McLeroy KR. Health promotion paradoxes, antinomies and conundrums: The importance of paradoxes. Health Educ Res 1996;11(1): i-xiii.

4. Poland B, Coburn D, Robertson A, Eakin J. Wealth, equity and health care: A critique of a "population health" perspective on the determinants of health. Soc Sci Med 1998;47(7):785-98.

5. Edwards N, Plotnikoff R, Spasoff R, et al. Multiple intervention effectiveness for the prevention of injuries: Development and testing of a decision support simulation framework. Poster presented at the 4th World Injury Prevention Conference. Amsterdam, 1998.

\section{Santé de la population : déterminants et interventions}

\author{
Nancy Edwards, IA, PhD
}

En tant que concept, la santé de la population est considérée comme un élément essentiel de la nouvelle Stratégie de la santé pour tous au $21^{\mathrm{e}}$ siècle. ${ }^{1}$ Cette nouvelle politique fait suite aux efforts menés pendant vingt ans pour atteindre les objectifs définis dans la Déclaration d'Alma Ata. Au cours de cette période de l'histoire de la santé publique au Canada, nous avons assisté à un déplacement des priorités en matière de services de santé communautaires qui, des interventions destinées à modifier le style de vie, ont évolué vers des programmes faisant appel aux grands principes de la promotion de la santé. Plus récemment, c'est la santé de la population qui a pris le devant de la scène en tant que concept à la base des programmes de santé publique. ${ }^{2}$ En s'appuyant sur l'expérience et les connaissances acquises grâce aux efforts de promotion de la santé et des styles de vie, la santé de la population est un concept qui focalise notre attention sur les inégalités dans l'état de santé de la population et sur leurs déterminants. À l'aube du troisième millénaire, l'un des grands défis que vont devoir relever ceux qui conçoivent, administrent et mettent en oeuvre les programmes de santé publique sera de trouver les moyens de s'attaquer effectivement à ces déterminants et à leurs interactions.

Par déterminants, il faut comprendre les causes, les facteurs à l'oeuvre et les influences contextuelles. De nombreux travaux de recherche ont porté sur les déterminants en tant que causes. L'épidémiologie et la médecine par exemple ont pour objectif de découvrir l'étiologie d'une maladie. Pour dégager ces causes, il faut comprendre très clairement le ou les mécanismes par lesquels un seul agent, une suite de facteurs ou un ensemble de circonstances peuvent aboutir à une maladie ou à une altération de l'état de santé. Les conclusions des

École de sciences infirmières, faculté des sciences de la santé, université d'Ottawa, ON études épidémiologiques sont souvent basées sur des modèles qui examinent les relations entre les facteurs de risque et la maladie. Ces modèles ne font qu'un tout petit peu la lumière sur les caractéristiques semblant offrir une protection contre la maladie. Toutefois, on peut en apprendre davantage sur ce qui protège contre la maladie en se servant des modèles qui examinent les liens de causalité entre les déterminants et la santé plutôt qu'entre les déterminants et la maladie. Cette façon de réfléchir à contre courant aux liens de cause à effet permet de mesurer d'autres variables et d'évaluer d'autres modèles statistiques. Des modèles théoriques (par ex., des modèles portant sur la résilience, le renforcement des capacités, la participation à la société et la cohésion sociale) sont nécessaires pour orienter ce type de réflexion.

Si de grands progrès ont été faits au plan de la découverte des mécanismes biologiques à la base des maladies, les interactions entre les déterminants sociaux et génétiques ainsi qu'entre les déterminants sociaux, environnementaux et physiques font beaucoup plus problème. Par exemple, bien que les interactions entre les effets combinés des expositions à un environnement physique tel que l'amiante ou la fumée ambiante soient bien documentées, nous commençons à peine à comprendre la synergie qui existe entre la pauvreté, la quasi absence de soutien social et le tabagisme. L'étude des paradoxes, définis en tant qu'observations qui contredisent notre compréhension actuelle des liens de cause à effet, ${ }^{3}$ nous encourage à remettre en cause nos hypothèses sur les populations à risque. Par exemple, quels sont les facteurs à l'origine de la résilience des jeunes qui vivent dans des familles ou des communautés à risque élevé? Pourquoi, en comparaison avec d'autres catégories, les taux de faible poids à la naissance sont-ils nettement plus faibles chez certaines catégories désavantagées au plan socio-économique? 
Par facteurs à l'oeuvre, on entend ceux qui ont pour effet de diminuer, renforcer ou modifier les liens entre deux facteurs ou plus. Ces facteurs ou déterminants peuvent être à l'origine des conditions nécessaires pour provoquer des interactions. Ils peuvent également influencer l'évolution des mécanismes de changement d'un état à un autre. De plus en plus, les transitions dans le cycle de la vie sont considérées comme autant de points d'intervention critiques. Il y a beaucoup à apprendre sur ces déterminants qui facilitent les transitions ou au contraire les rendent plus difficiles.

Le contexte fait référence au milieu, à la situation ou au scénario dans lequel les déterminants vont se manifester comme causes ou facteurs à l'oeuvre. Au niveau macro de l'échelle, les influences contextuelles concernent les grands facteurs propres au système comme les influences politiques (par ex., un changement de gouvernement) et les forces économiques mondiales (par ex., l'effondrement du marché asiatique). Ces influences s'exercent en général de façon plus subtiles sur notre vie quotidienne. Toutefois, elles constituent une force permanente qui façonne nos valeurs, nos opinions et nos attentes. À l'autre extrême, les micro-déterminants sont ceux qui influencent de façon délibérée notre quotidien. Ainsi, les lieux de vie dans lesquels nous passons notre temps (lieu de travail, foyer) et les contacts sociaux que nous y avons ont une influence sur notre état de santé relatif, bon ou mauvais. Dans leur critique des déterminants sociaux tels que les considère l'Institut canadien de recherches avancées, Poland et al. ${ }^{4}$ avancent que les grandes forces politiques et sociales derrière les déterminants de la santé (par ex., la mondialisation et la structure de classes) justifient une analyse plus complète.

En fin de compte, la recherche sur les déterminants a pour objectif d'orienter les modalités d'intervention. Une meilleure compréhension des déterminants, de leurs interactions et des influences contextuelles devrait permettre de mieux calibrer les programmes de santé publique et donc leurs répercussions. Toutefois, les difficultés qu'entraîne une pareille démarche résident dans la complexité des déterminants et également dans leur dynamique qui a pour effet de changer en permanence les condi- tions d'intervention. Donc, une perspective axée sur les déterminants fait ressortir le besoin de programmes pluriels conçus pour établir des synergies potentielles entre les stratégies d'intervention. Pour les planificateurs des programmes, cela soulève de nombreuses questions sur les combinaisons optimales et l'ordre des multiples interventions; sur les contributions relatives des interventions pluriels; sur l'impact de leur fréquence, leur intensité, leur interdépendance et leur différenciation; ainsi que sur la nature répétitive de l'impact des multiples interventions (en série, en parallèle et avec décalage). Il conviendra de mettre au point de nouveaux outils et stratégies à l'appui des décisions pour aider les administrateurs de programmes à faire face à ce niveau de complexité sans précédent dans la conception des programmes. ${ }^{5}$ C'est LE défi qui est posé à la santé de la population; comment se servir des découvertes qui apparaissent au sujet des déterminants et de leurs interactions pour orienter l'élaboration de la prochaine génération des programmes de santé publique.

\section{International Travel and Health (1999) Vaccination Requirements and Health Advice \\ World Health Organization Order \#1189900 \\ $\$ 21.42$

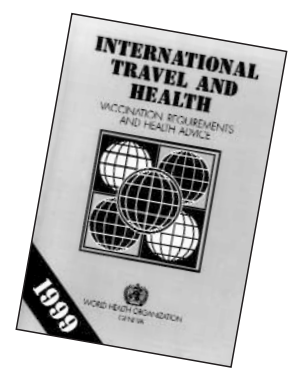 \\ This booklet is addressed to national health administrations and to the practising physicians, tourist agencies, shipping companies, airline operators, and other bodies who are called upon to give health advice to travellers. In addition to summarizing the vaccina- tion requirements of individual countries, the booklet indicates the main areas where malaria transmission occurs and where Plasmodium falciparum is resistant to drugs. Other chapters cover certain health hazards to which the traveller may be exposed and indicate the areas in which these hazards are most likely to occur. The booklet also recommends a number of pre- cautions that the wise traveller should take when visiting unfamiliar places. \\ Available from CPHA, Tel: 613-725-3769, Fax: 613-725-9826

\begin{abstract}
Voyages internationaux et santé (1999) Vaccinations exigées et conseils d'hygiène

Organisation mondiale de la santé № de commande 2189900

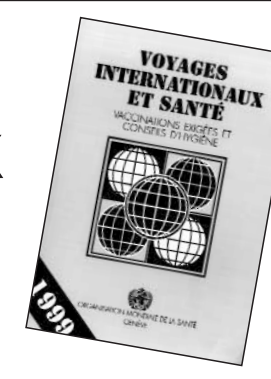

$21,42 \$$

La présente publication s'adresse aux administrations de la santé, au corps médical, aux agences de tourisme, aux compagnies maritimes et aériennes et aux autres organismes qui sont amenés à donner des conseils d'hygiène aux voyageurs. En plus du résumé des exigences des pays en matière de vaccinations, la publication indique les principales zones où il y a transmission du paludisme et résistance de Plasmodium falciparum aux médicaments. D'autres chapitres décrivent certains risques pour la santé des voyageurs et indiquent les régions où ces risques se rencontrent le plus souvent. La publication recommande aussi certaines précautions que le voyageur serait avisé de prendre lorsqu'il se rend dans des régions peu connues.
\end{abstract} \\ Disponible au l'ACSP, Tél. : 613-725-3769, Télec. : 613-725-9826}

